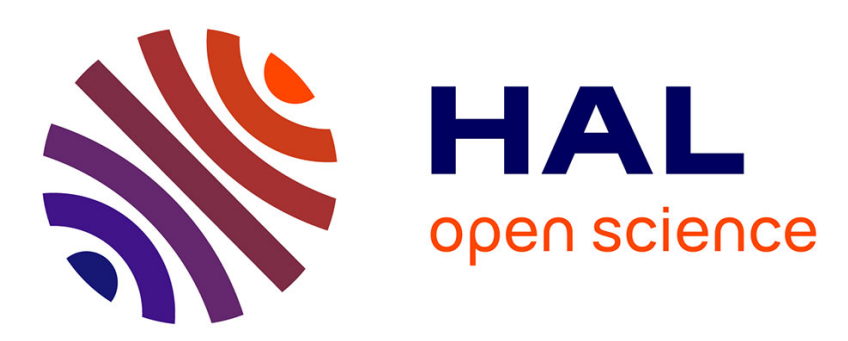

\title{
Useful applications of the electron localization function in high pressure crystal chemistry
}

Julia Contreras-García, A.M. Ángel M. Pendás, Bernard Silvi, J. Manuel

Recio

\section{- To cite this version:}

Julia Contreras-García, A.M. Ángel M. Pendás, Bernard Silvi, J. Manuel Recio. Useful applications of the electron localization function in high pressure crystal chemistry. Journal of Physics and Chemistry of Solids, 2009, 69 (9), pp.2204. 10.1016/j.jpcs.2008.03.028 · hal-00560686

\section{HAL Id: hal-00560686 \\ https://hal.science/hal-00560686}

Submitted on 29 Jan 2011

HAL is a multi-disciplinary open access archive for the deposit and dissemination of scientific research documents, whether they are published or not. The documents may come from teaching and research institutions in France or abroad, or from public or private research centers.
L'archive ouverte pluridisciplinaire HAL, est destinée au dépôt et à la diffusion de documents scientifiques de niveau recherche, publiés ou non, émanant des établissements d'enseignement et de recherche français ou étrangers, des laboratoires publics ou privés. 


\section{Author's Accepted Manuscript}

Useful applications of the electron localization function in high pressure crystal chemistry

Julia Contreras-García, Ángel M. Pendás, Bernard Silvi, J. Manuel Recio

PII: S0022-3697(08)00104-2

DOI: doi:10.1016/j.jpcs.2008.03.028

Reference: PCS 5435

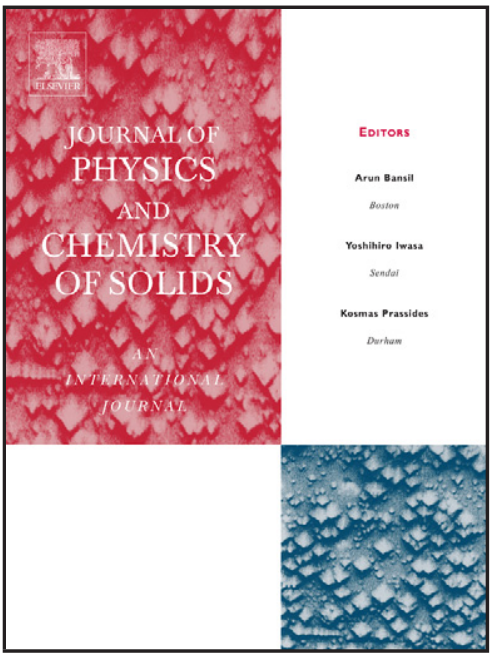

www.elsevier.com/locate/jpcs

To appear in: Journal of Physics and Chemistry of Solids

Cite this article as: Julia Contreras-García, Ángel M. Pendás, Bernard Silvi and J. Manuel Recio, Useful applications of the electron localization function in high pressure crystal chemistry, Journal of Physics and Chemistry of Solids (2008), doi:10.1016/j.jpcs.2008.03.028

This is a PDF file of an unedited manuscript that has been accepted for publication. As a service to our customers we are providing this early version of the manuscript. The manuscript will undergo copyediting, typesetting, and review of the resulting galley proof before it is published in its final citable form. Please note that during the production process errors may be discovered which could affect the content, and all legal disclaimers that apply to the journal pertain. 


\title{
Useful Applications of the Electron Localization Function in High Pressure Crystal Chemistry
}

\author{
Julia Contreras-García, ${ }^{a}$ Ángel M. Pendás, ${ }^{a}$ Bernard Silvi, ${ }^{\text {b }}$ and J. Manuel Recio ${ }^{\text {a }}$ \\ ${ }^{a}$ Departamento de Química Física y Analítica, Universidad de Oviedo, E-33006 Oviedo, Spain \\ ${ }^{b}$ Laboratoire de Chimie Théorique, Université Pierre et Marie Curie, F-75252Paris cédex, France
}

\begin{abstract}
The main features of a new computational code aimed at the topological analysis of the Electron Localization Function (ELF) in crystals are hereby presented. Besides the complete localization of all critical points, the code is able to determine the limiting surfaces which define the chemical regions associated with the so-found maxima (or attractors) of the ELF. Hence, integrations of density operators within these basins provide charges and volumes to cores, bonds, and lone pairs. Two illustrative applications have been selected. Firstly, we examine the $\mathrm{Z}$ dependence of basin compressibilities in the elements of the first two rows of the Periodic Table, and secondly, we focus our attention on the chemical changes across the pathway of the zinc blenderocksalt phase transition in $\mathrm{BeO}$.
\end{abstract}

Keywords: A. Elements; C. Ab-initio calculation; C. High pressure; D. Equation of state; D. Phase transitions

Corresponding author: Julia Contreras García. Tel.: +34985102991. Fax: +34985103125

E-mail address: julia@carbono.quimica.uniovi.es 


\section{Introduction}

Due to the high coordination indexes and to the numerous atom-atom contacts, the presence of different bonding types is a common and genuine feature of solids which hampers their characterization when compared with the molecular realm. Performing a rigorous qualitative and quantitative classification of these bonds, based on the same theoretical approach, has revealed itself a challenge and a source of debate [1]. The widest-spread method of approach has been to consider the topological analysis of the electron density (AIM) as developed by Bader and co-workers [2,3], according to which the existence of a bond is associated to the presence of a bond path in the stable structure of the solid.

Besides, it has been long recognized that further insight into the nature of the bond can be gained by resorting to the electron localization function (ELF) [4]. The ELF was introduced in 1990 by Becke and Edgecombe [5] in an attempt to analyse electron localization from a theoretical standpoint. Later on, the work carried out by Silvi and Savin [6], and the formulation of the latter [7] within the DFT framework have given value to the ELF in solid state science. Within this formalism, the ELF can be understood as a local measure of the excess of local kinetic energy of electrons due to the Pauli principle. It is expressed in terms of the local kinetic energy $(t)$, its value for this density in a bosonic system $\left(t_{\mathrm{W}}\right)$ and the kinetic energy of the homogeneous electron gas ( $t_{\mathrm{TF}}$, Thomas-Fermi):

$$
\eta=\left(1+\frac{t(r)-t_{W}(r)}{t_{T F}(r)}\right)^{-1}
$$


For convenience reasons it is defined in such a way that it runs from 0 to 1 . It approaches 1 in those regions of space where localization is high, and tends to a value of 0.5 when the density distribution approaches to homogeneity. Within the context of our work, it is also interesting to remark that the ELF topological analysis allows a partition of the 3dimensional space in terms of non-overlapping regions with a full chemical meaning: electronic shells, bonds and lone pairs. This ability makes the ELF be an appropriate formalism to reconcile the traditional chemical language with the outcome of quantummechanical calculations.

However, the application of the ELF formalism in high-pressure condensed matter has been scarce due to major computational difficulties imposed by periodicity $[4,8,9]$. Our work has been focused to the development of a computational code attempting to fulfil the gap existing between the ELF topological analyses of bonding in the molecular and the crystalline realms. Localization of ELF critical points, evaluation of charges and volumes of basins, and visualization of interesting chemical entities within the ELF are some of the possibilities offered by our new code. A brief description of the main tasks of the program, examples of bonding analyses in prototypical solids, and selected applications in highpressure crystal chemistry are presented below.

\section{The program}

The program is currently performing three different ELF-related tasks once an all-electron crystalline wave function is introduced as input. The process is as follows. Firstly, the code searches the complete set of critical points within the cell. The irreducible Wigner Seitz cell 
is constructed thereto, and its topological properties are applied in order to localize all the critical points by means of recursive algorithms. Due to the great concentration of critical points around the cores, and their steep profile, the result is refined by a spherical search around the nuclei at the shell radii distances.

The analysis of the ELF profile even allows us to identify the relationship between basins, and hence to characterize the weak interactions through the localization of the bond points between the basins. The relative value of ELF at those points sheds light into the "separation" between them, so that the higher the value of ELF at those points (so called, bip, bond interaction points), the less separated they are. Hence, a complete analysis of the attractors provides insight into the strong interactions, and a subsequent analysis of the bond points between them enables the characterization of the weak ones. The localization of the complete set of critical points as performed by our code enables in most cases the automatic localization of the bip. Thus, both the relationship between basins and the weak interactions can be efficiently identified, a task that becomes increasingly difficult as the complexity of the solid structure grows, if we follow a visual approach (most common method up to now).

Last but not least, the localization of the complete set of critical points not only provides a clear and quantitative view of the bonding in the crystal: as already found in the AIM theory of solids, the use of the cage points as vertices drives back to the common inorganic description of crystals based on polyhedra. This splitting provides a theoretical root for this description as well as, and most importantly, theoretical radii along different directions for the elements and their shells. 
Secondly, and once the attractors have been identified, the program is able to locate the boundary limit of the region of space associated to each of them, the basin, and therefore, it is able to calculate the integrals of any density operator provided. Currently implemented basin properties are charge population, volume and momenta. Work is in progress for the implementation of localization/delocalization indexes [10] and probabilities [11]. Some representative results for different kinds of solids are summarized below.

Finally, visualization of the net of critical points, basins and surfaces can be obtained if desired by demanding the construction of 1, 2 and 3-dimensional grids. Several common visualization formats are available (Gnuplot, Gaussian cube, etc.).

\section{Molecular, ionic, covalent, and metallic solids}

As shown in Figure 1, ELF is able to clearly represent and differentiate between the different types of solids. Carbon dioxide, as a molecular solid, shows well differentiated chemical ELF objects that are identified as $\mathrm{CO}_{2}$ molecules. The carbon core (K-shell) in the middle, $\mathrm{C}(\mathrm{C})$, with the expected charge of two electrons, a general picture that is reproduced in all the cases and that is not affected by the environment: cores approach the nominal value of two electrons [12]. Going then along the $\mathrm{CO}_{2}$ molecular axis, we first find the basins corresponding to a double bond, $\mathrm{B}(\mathrm{C}-\mathrm{C})$. Notice that it is the number of basins that determines the multiplicity and not the number of electrons per bond. Finally, we clearly identify the oxygen core, $\mathrm{C}(\mathrm{O})$, and its lone pairs, $\mathrm{LP}(\mathrm{O})$. Analysing the charge distribution one realizes that not only is the Lewis picture recovered, but that a weight is 
given to the different resonant forms.

A covalent solid like diamond displays a continuous 3D-network of tetrahedrically coordinated carbons, bound together by means of single-bond basins, $\mathrm{B}(\mathrm{C}-\mathrm{C})$. In this case, perfect electron pairs are found, as expected from the perfect covalency of the compound (no resonant forms). Ionic compounds like $\mathrm{LiF}$ are characterized by the absence of bond basins, only showing closed-shell basins ( $\mathrm{K}$ and L, C(Li), C(F), and V(F)) whose shape approach that of a sphere and whose charge corresponds to that of a complete transfer.

As far as metals are concerned, their ELF profile is nearly flat as represented by the surface surrounding Na cores (K and L shells), a surface that resembles the traditional electron gas model of metals. However, this jellium is not at all homogeneous, but it presents numerous maxima, $\mathrm{B}(\mathrm{Na}-\mathrm{Na})$ [8], as already observed in the density topological analysis of other metals [13]. It must be highlighted that these maxima, to which a low population is attributed, are in fact distributed in the cell in such a way that the positions of the interstitial attractors are close to the positions of anions in ionic compounds. In other words, the electrons behave as electrides towards whose positions electronegative atoms head to form anions in ionic crystals.

\section{Chemical partition of compressibility}

Our target systems are the crystalline phases of the elements of the first two rows of the Periodic Table. The electronic structure calculations have been carried out in the framework of the density functional theory (DFT) implemented in the CRYSTAL code 
[14]. A number of pressure (p)-volume (V) points have been calculated for each of the compounds, and the topological analysis of the ELF was performed with the new code in order to evaluate the rate at which the volumes of the basins decrease when pressure is applied to them. As explained above, two different types of attractors are to be found: atomic shells and bonding attractors. In analogy with the jellium model, these will be resumed in core (inner shells) and valence (electrons coming from the last shell).

We have found that it is always the valence that is the main responsible for the bulk compression in all the crystalline systems, whereas cores remain practically untouched. As far as valence behavior is concerned, there is one main factor to be taken into account: Pauli repulsion induced by pressure. This force arises from the enhanced overlaps among localized basins upon crystal compression, and its amount depends both on the overlapping, which increases with the proximity, and on the ELF value of the basins. According to these simple ideas, the periodic trends observed in Fig. 2 (the compressibility decreases along $\mathrm{Li}$, Be, C; Ne, Na, Mg; and increases for Li-Na and Be-Mg) can be razionalized as follows. ELF basins in noble elements are well separated. In these crystals, the negligible overlap between basins allows an easy reduction of the volume as pressure is applied, and, therefore, a great compressibility is expected for this kind of compounds. A completely different picture is displayed by very low compressible compounds, such as diamond, where the basins show high ELF values separated by steep valleys. Hence, there are no large interstices without localized pairs, and the increase of energy induced by pressure prevents volume reduction. Metals represent a scenario in between: the ELF valence profile is a flat platform with intermediate localization values (see Fig. 1), and the bulk compressibility of metals is, therefore, lower than in crystals of noble elements, but higher 
than in covalent compounds. Furthermore, the order of compressibilities within compounds of the same nature also follows this trend, so lithium is more compressible than berillium since its ELF platform is around 0.27 , whereas that of berillium is around 0.61 .

Quantitative data of the basin response to pressure is obtained through equations of state (EOS) fittings. This is of critical importance in material design and earth studies and, therefore, it becomes interesting to find out that compressibility can also be expressed as a sum of chemically meaningful basins, just as in the case of AIM [15]:

$$
\kappa=-\frac{1}{V}\left(\frac{\partial V}{\partial p}\right)=\sum_{i} f_{i} \kappa_{i}, \quad \frac{1}{\kappa_{i}}=-V_{i}\left(\frac{\partial p}{\partial V_{i}}\right)
$$

where $f_{i}=V_{i} / \bar{V}$ is the fractional occupation of the basin in the unit cell. In all the cases we have analysed, the valence basins obey standard EOS as the Vinet one, but the cores do not, probably due to the high electron density enclosed in these small basins and/or to higher precision requirements.

EOS parameters, i.e. bulk moduli and its first pressure derivative, both evaluated at zero pressure, were determined for the ELF basins of the elements discussed above, as well as for core, valence and bond basins involved in several simple and representative compounds such as $\mathrm{LiF}$ (ionic), $\mathrm{N}_{2}$ and $\mathrm{CO}_{2}$ (molecular), $\mathrm{BeO}$ (polar), etc. The value of the crystal compressibility in the solid is well reproduced by the sum of the basin contributions (errors within 1-3\%). It can be seen from the equation above that two factors determine the 
contribution of the different basins to the total crystalline compressibility: on the one hand the relative volume and, on the other hand, the basin compressibility. Due to both factors, the main contribution is associated to valence which is both bigger and more compressible. For example, the valence compressibility in $\mathrm{Li}$ is $7.56 \times 10^{-2} \mathrm{GPa}^{-1}$ and the calculated crystal value is $7.92 \times 10^{-2} \mathrm{GPa}^{-1}$. The contribution from the K-shell of $\mathrm{Li}$ is almost negligible since its charge/volume ratio is very high and strong forces are acting within it. The same applies to binary ionic and polar compounds such as $\mathrm{LiF}$ and $\mathrm{BeO}$. We have calculated, for instance, that the oxide valence (the only valence basin) accounts for $99.2 \%$ of the $\mathrm{BeO}$ crystalline compressibility.

Molecular crystals, given their more complex pattern, can also illustrate how the compressibility and the chemical nature of the basins are linked. Thus, in $\mathrm{N}_{2}$ the contributions to the crystal compressibility are mainly due to the lone pairs (98.1\%) but the basin compressibilities do not follow such a clear sequence as in the previous examples $\left(\mathrm{k}_{\text {core }}=1.40 \times 10^{-3} \mathrm{GPa}^{-1}, \mathrm{f}_{\text {core }}=0.024, \mathrm{k}_{\text {bond }}=1.12 \times 10^{-3} \mathrm{GPa}^{-1}, \mathrm{f}_{\text {bond }}=0.136, \mathrm{k}_{\text {lonepair }}=1.82 \times 10^{-2}\right.$ $\mathrm{GPa}^{-1}, \mathrm{f}_{\text {lonepair }}=0.862$ ), where all the regions were atomic shells. It is found that the compressibility of the core is slightly greater than that of the bond. This is not surprising if one realizes that in both cases the volume in which electrons are localized is very small, but the populations of core and bond are 2.08 and 3.51 electrons, respectively. In both cases electrons are subjected to very strong electrostatic repulsions, but in the second case, also strong Pauli repulsions are taking place. These results are very promising and need to be extended to more complex materials to derive general conclusions, though it is very reasonable to assume that the basic ideas can be already found in these simple compounds. 


\section{B3-B1 pathway in BeO: energy profile, structural changes and ELF evolution.}

The next step after the application of the new code to the microscopic interpretation of EOS in solids is to examine whether the ELF also provides an insight into chemical changes. With this aim in mind, the pressure-induced phase transition from the zinc-blende (B3) to the rocksalt phase (B1) of $\mathrm{BeO}$ has been studied. Both literature surveys [16-18] and present computations have shown that the orthorhombic Imm2 space group is energetically favoured for the description of the transition, with a barrier of $0.51 \mathrm{eV}$ at the transition pressure of $114 \mathrm{GPa}$ according to our calculations. Under this symmetry, the z coordinate of $\mathrm{Be}, \mathrm{z}_{\mathrm{Be}}$, evolves from 0.25 in the $\mathrm{B} 3$ to 0 in the $\mathrm{B} 1$ phase, and can be taken as the transition coordinate. The energetic profile of the transition is found to proceed in just one step whose maximum is located at around $\mathrm{z}_{\mathrm{Be}}=0.125$. Theoretical studies usually complement the energetic analysis by identifying atomic displacements and changes in distances (Figure 2b, upper curves) and coordination. In the case of study, the coordination of Be goes from a 4-fold tetrahedron to a 6-fold octahedron.

Just as for the compressibility, the chemical and structural changes taking place across the transition are merely reflected by the valence. Analysis of the ELF topology of the oxygen L shell, $\mathrm{V}(\mathrm{O})$, reveals that it is initially divided into four different basins $(2+2)$ whose attractors point at the four coordinated Be. It is only after the transition path has been traced up to around $\mathrm{z}_{\mathrm{Be}}=0.15$, that two new $\mathrm{O}$ valence attractors appear. This fact has a catastrophic nature and reveals the onset of the new phase. The formation of the two new basins brakes the increasing in energy associated with the transformation. Furthermore, these new oxygen L-shell basins evolve along the transition in such a way that they 
resemble the other four, and the valence is finally divided into six equivalent basins, which perfectly show the change in coordination occurring in the crystal. Hence, this topological change drives us back to both approaches of the phase transition, the energetic and the structural one.

\section{Summary and some prospects.}

The electron localization function has proved to be a very useful tool in analysing the Lewis picture of molecules, and more specifically, the nature of the bonds. Due to computational difficulties, its application in solids has until now been scarce and mainly qualitative. A new code is hereby presented which widens the application of the ELF topological analysis to all kinds of solids, allowing a microscopic interpretation of the physical and chemical changes that pressure induces upon their bonds. The integration of the ELF basins as performed by our code enables the calculation of electron shell, bond and lone pair basin compressibilities of a large variety of crystalline systems. Data obtained so far do correlate with the chemical concepts as classically established. This does not only confirm these principles, but also endows them with a theoretical background enabling future behaviour foreseeing.

Our next step towards a deeper understanding of the processes taking place within solids is to carry out the analysis of phase transitions where a change in the nature of the bond occurs. Work is in progress in both $\mathrm{CO}_{2}$ and $\mathrm{SiO}_{2}$, where the change from molecular to 
covalent and polar-ionic networks awakes much interest due to the natural relevance of both compounds [19-21]. In order to provide a non-ambiguous tool to understand these changes in the bond nature, we are currently working in the development of a user-friendly index that would quantify the character of the bond based on the ELF topological analysis $[1,22]$.

\section{Acknowledgements.}

Financial support from the Spanish MEC and FEDER program under projects MAT200613548-C02-02 and CTQ2006-02976 are gratefully acknowledged. JCG thanks the Spanish MEC for a FPU postgraduate grant and for funding her stay at the Laboratoire de Chimie Theorique (Universite Pierre et Marie Curie, Paris) where part of this work has been done.

\section{References}

[1] P. Mori-Sánchez, A. Martín Pendás, V. Luaña, J. Am. Chem. Soc 124 (2002) 14721

[2] R.F.W. Bader; Atoms in Molecules: A. Quantum Theory; Oxford University. Press: Oxford, U.K., 1990.

[3] A. M. Pendas, A. Costales, V. Luaña, Phys. Rev. B 55 (1997) 4275.

[4] C. Gatti, Zeit. Kristallogr. 220 (2005) 399.

[5] A.D. Becke, K.E. Edgecombe, J. Chem. Phys. 92 (1990) 5397.

[6] B. Silvi , A. Savin, Nature. 371 (1994) 683.

[7] A. Savin, O. Jepsen, J. Flad, L.K. Andersen, H. Preuss, H.G. von Schnering, Angew. Chem. Int. Ed. Engl. 31 (1992) 187.

[8] B. Silvi, C. Gatti, J. Phys. Chem. 104 (2000) 947.

[9] A. Savin, J. Phys. Chem. Sol 65 (2004) 2025

[10] A. Savin, B. Silvi, F. Colonna, Can. J. Chem. 74 (1996) 1088. 
[11] E. Chamorro, P. Fuentealba, A. Savin, J. Comput. Chem. 24 (2003) 496

[12] M. Kohout, A. Savin, Int. J. Quant. Chem. 60 (1996) 875.

[13] A. M. Pendás, M. A. Blanco, A. Costales, P. Mori-Sánchez, V. Luaña, Phys. Rev. Lett. 83 (1999) 1930.

[14] V. R. Saunders, R. Dovesi, C. Roetti, M. Causa, N. M. Harrison, R. Orlando, C. M. Zicovich-Wilson, CRYSTAL98 User’s manual, University of Torino, Torino (1998).

[15] A.M. Pendás, A. Costales, M.A. Blanco, J.M. Recio, V. Luaña, Phys. Rev. B 62 (2000) 13970

[16] M. S. Miao, W. R. L. Lambrecht, Phys. Rev. Lett. 94 (2005) 225501

[17] D. M. Hatch, H. T. Stokes, J. Dong, H. Wang, J. P. Lewis, Phys. Rev. B 71 (2005) 184109.

[18] J. C. Boettger, J. M. Wills, 54 (1996) 8965.

[19] V. Iota, C-S. Yoo, Z. Jenei, J. Park-Kepleis, H. Cynn, W. Evans, Nature Materials 6 (2007) 34

[20] S. Serra, C. Cavazzoni, G. L. Chiarotti, S. Scandolo, E. Tosatti, Science 284 (1999) 788.

[21] S. Jenkins, J. Phys: Condens. Matter, 14 (2002) 10251

[22] P. Macchi, A. Sironi, Coordination Chem. Rev. 239 (2003) 383 


\section{FIGURE CAPTIONS}

Fig. 1. From left to right, molecular $\mathrm{CO}_{2}$, C-diamond, rocksalt $\mathrm{LiF}$ and bcc Na. Labels stand for the name and the charge of each basin.

Fig. 2. Relative volumes vs. pressure of some of the elements of the first two rows of the periodic table. Curves at the top correspond to cores, with the same symbols as the valence curves at the bottom.

Fig. 3. Evolution of the six Be-O shortest distances (upper curves) and the distances from oxygen nucleus to its valence attractors (bottom) along the transition pathway. X stands for Be or the corresponding attractor. The black circle marks the emergence of the new oxygen basin. Numbers in brackets stand for the multiplicities associated with the curves. 
Fig.1

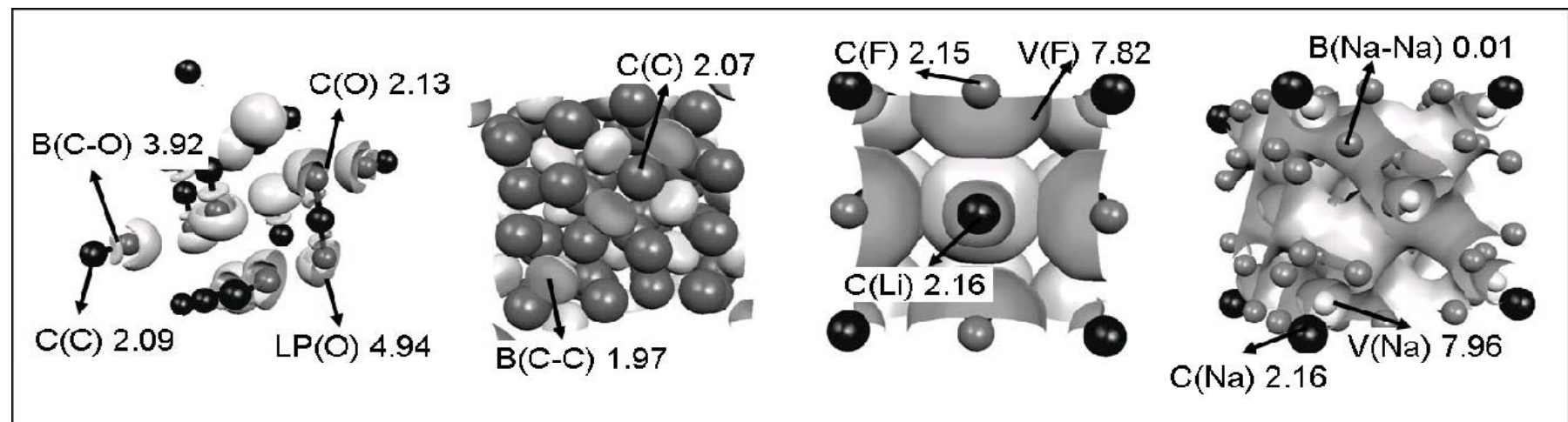

Fig. 2

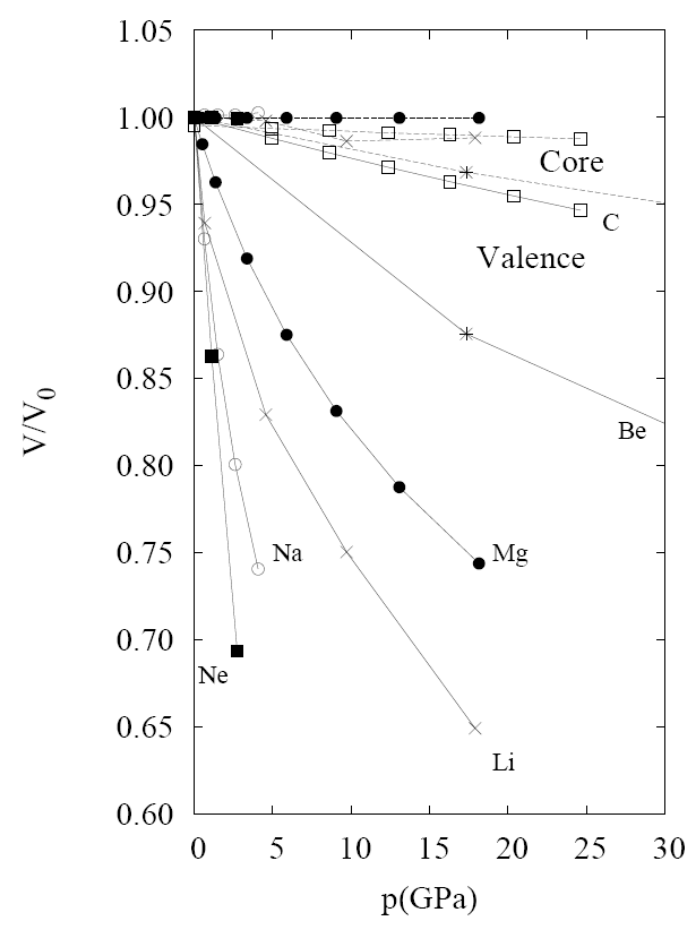


Fig. 3

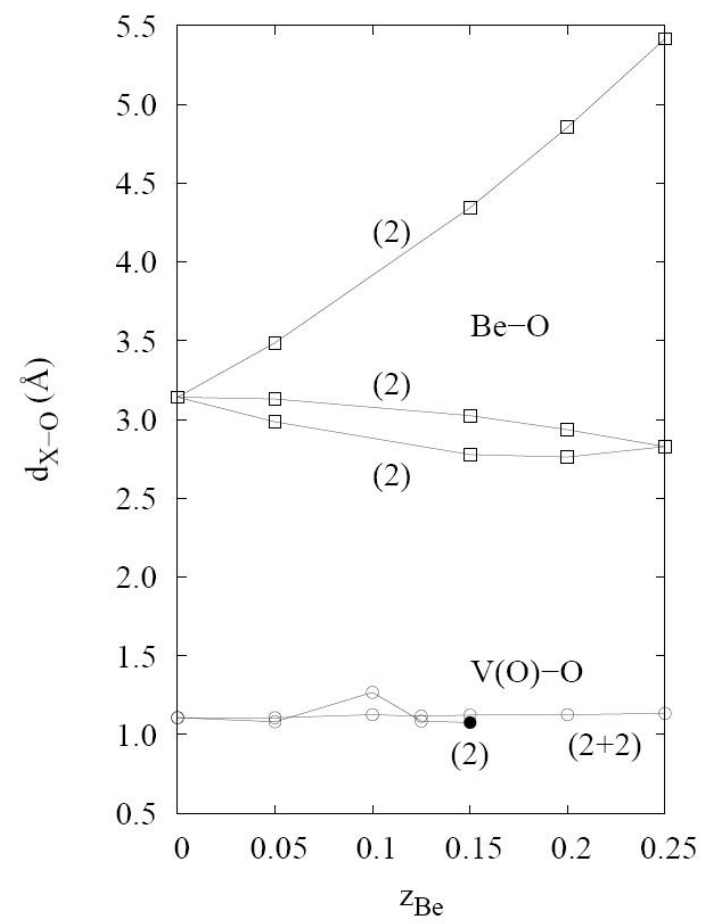

